\title{
Ten-electron Coordination and Reactivity of an Arylphosphinidene Ligand
}

\author{
M. Esther García, Víctor Riera, Miguel A. Ruiz*, David Sáez, Hayrullo Hamidov, ${ }^{\dagger}$ John C. Jeffery ${ }^{\dagger}$ and \\ Thomas Riis-Johannessen, ${ }^{\dagger}$ \\ Departamento de Química Orgánica e Inorgánica/IUQOEM, Universidad de Oviedo, 33071 Oviedo, Spain. ${ }^{\dagger}$ School of \\ Chemistry, University of Bristol, Bristol BS8 ITS, UK.
}

\section{Supporting Information}

Preparative Procedures and Microanalytical Data for New Compounds. $\left(\mathrm{R}^{*}=2,4,6-\mathrm{C}_{6} \mathrm{H}_{2}{ }^{\mathrm{t}} \mathrm{Bu}_{3}\right)$

\section{General Procedures and Starting Materials}

General Comments. All manipulations and reactions were carried out under a nitrogen $(99.9995 \%)$ atmosphere using standard Schlenk techniques. Solvents were purified according to literature procedures (1) and distilled prior to use. Petroleum ether refers to that fraction distilling in the range $65-70{ }^{\circ} \mathrm{C}$. Compound $\left[\mathrm{Mo}_{2} \mathrm{Cp}_{2}(\mu-\mathrm{PR} *)(\mathrm{CO})_{4}\right]\left(\mathrm{Cp}=\eta^{5}-\mathrm{C}_{5} \mathrm{H}_{5}\right.$; $\left.\mathrm{R}^{*}=2,4,6-\mathrm{C}_{6} \mathrm{H}_{2}{ }^{\mathrm{t}} \mathrm{Bu}_{3}\right)(2)$ and $\left[\mathrm{H}\left(\mathrm{OEt}_{2}\right)_{2}\right]\left[\mathrm{BAr}_{4}{ }_{4}\right]\left\{\mathrm{Ar}^{\prime}=3,5-\mathrm{C}_{6} \mathrm{H}_{3}\left(\mathrm{CF}_{3}\right)_{2}\right\}$ (3) were prepared as described previously. All other reagents were obtained from the usual commercial suppliers and used as received. Photochemical experiments were performed using jacketed quartz Schlenk tubes, cooled by tap water (ca. $15^{\circ} \mathrm{C}$ ). A $400 \mathrm{~W}$ mercury lamp (Applied Photophysics) placed ca. $1 \mathrm{~cm}$ away from the Schlenk tube was used for these experiments. Chromatographic separations were carried out using jacketed columns cooled by tap water. Commercial aluminium oxide (activity I, 150 mesh) was degassed under vacuum prior to use. The latter was mixed under nitrogen with the appropriate amount of water to reach the activity desired. Carbonylation experiments were carried out using Schlenk tubes equipped with Young's valves. Filtrations were performed using diatomaceous earth. IR CO stretching frequencies were measured in solution and are referred to as $v_{\mathrm{CO}}$ (solvent). Nuclear Magnetic Resonance (NMR) spectra were routinely recorded at 300.13 $\left({ }^{1} \mathrm{H}\right), 121.50\left({ }^{31} \mathrm{P}\left\{{ }^{1} \mathrm{H}\right\}\right)$ or $75.47 \mathrm{MHz}\left({ }^{13} \mathrm{C}\left\{{ }^{1} \mathrm{H}\right\}\right)$ at $290 \mathrm{~K}$ in $\mathrm{CD}_{2} \mathrm{Cl}_{2}$ solutions unless otherwise stated. Chemical shifts $(\delta)$ are given in ppm, relative to internal tetramethylsilane (TMS) or external $85 \%$ aqueous $\mathrm{H}_{3} \mathrm{PO}_{4}$ solutions $\left({ }^{31} \mathrm{P}\right)$. Coupling constants $(J)$ are given in Hertz. Assignment of the ${ }^{13} \mathrm{C}$ NMR resonances for the aryl group follow the labelling shown in the figure below, and are reported as ${ }^{n} \mathrm{C}\left(\mathrm{C}_{6} \mathrm{H}_{2}\right)$ or ${ }^{\mathrm{m}} \mathrm{C}\left({ }^{\mathrm{t}} \mathrm{Bu}\right)$ as required $(\mathrm{n}=1$ to $6 ; \mathrm{m}=1,2)$.

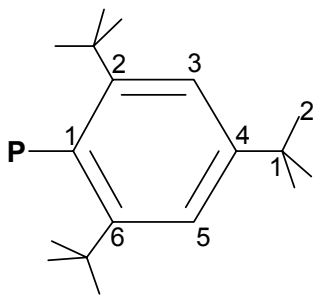

Preparative procedures, spectroscopic and microanalytical data for the new compounds

Preparation of $\quad\left[\mathrm{Mo}_{2} \mathrm{Cp}_{2}\left(\mu-\kappa^{1}: \kappa^{1}, \eta^{6}-\mathrm{PR}^{*}\right)(\mathrm{CO})_{2}\right] \quad$ (3). A tetrahydrofuran solution $(15 \mathrm{~mL})$ of the compound $\left[\mathrm{Mo}_{2} \mathrm{Cp}_{2}(\mu-\right.$ $\left.\left.\mathrm{PR}^{*}\right)(\mathrm{CO})_{4}\right](0.500 \mathrm{~g}, 0.704 \mathrm{mmol})$ was placed in a quartz jacketed Schlenk tube refrigerated by tap water and irradiated with UV-visible light for $4 \mathrm{~h}$, whilst slowly bubbling nitrogen (99.9995\%) through the solution. Solvent was then removed in vacuo and the residue was dissolved in the minimum of dichloromethane and chromatographed on an alumina column (Activity IV, $30 \times 3 \mathrm{~cm}$ ) at $288 \mathrm{~K}$. Elution with petroleum ether gave first trace amounts of the starting complex and $\left[\mathrm{Mo}_{2} \mathrm{Cp}_{2}(\mu-\mathrm{H})\left\{\mu-\mathrm{P}\left(\mathrm{CH}_{2} \mathrm{CMe}_{2}\right) \mathrm{C}_{6} \mathrm{H}_{2}{ }^{t} \mathrm{Bu}_{2}\right\}(\mathrm{CO})_{4}\right]$ (2). Elution with a dichloromethane:petroleum ether (1:3) mixture gave a yellow fraction. Removal of solvents in vacuo from the latter fraction gave compound 3 as a brown microcrystalline solid $(0.085 \mathrm{~g}, 19 \%)$. Elution with a dichloromethane:petroleum ether (1:1) mixture gave a brown fraction. Removal of solvents in vacuo from the latter fraction gave $\left[\mathrm{Mo}_{2} \mathrm{Cp}_{2}(\mu\right.$ $\left.\left.\mathrm{PR}^{*}\right)(\mu-\mathrm{CO})_{2}\right](0.185 \mathrm{~g}, 41 \%)$ as a dark-brown solid. The crystals of compound 3 used in the X-ray study were grown by slow diffusion of petroleum ether into a toluene solution of the complex, at $253 \mathrm{~K}$. Analysis calculated for $\mathrm{C}_{30} \mathrm{H}_{39} \mathrm{Mo}_{2} \mathrm{O}_{2} \mathrm{P}(3)$ : C, 55.05; H, 6.01. Found: $\mathrm{C}$, 54.85; H, 6.10. $v_{\mathrm{CO}}\left(\mathrm{CH}_{2} \mathrm{Cl}_{2}\right): 1891$ (vs), 1816 (s) $\mathrm{cm}^{-1} .{ }^{1} \mathrm{H}$ NMR: $\delta 5.59$ $\left(\mathrm{s}, 2 \mathrm{H}, \mathrm{C}_{6} \mathrm{H}_{2}\right), 5.37(\mathrm{~s}, 5 \mathrm{H}, \mathrm{Cp}), 5.29\left(\mathrm{~d}, J_{\mathrm{HP}}=2,5 \mathrm{H}, \mathrm{Cp}\right), 1.31(\mathrm{~s}, 18 \mathrm{H}, 2$ $\left.\mathrm{x}{ }^{\mathrm{t}} \mathrm{Bu}\right), 1.18$ (s, 9H, $\left.{ }^{\mathrm{t}} \mathrm{Bu}\right) \cdot{ }^{31} \mathrm{P}\left\{{ }^{1} \mathrm{H}\right\}$ NMR: $\delta 509.9$ (s, $\left.\mu-\mathrm{PR} *\right) .{ }^{13} \mathrm{C}\left\{{ }^{1} \mathrm{H}\right\}$ NMR (100.63 MHz): $\delta 242.2\left(\mathrm{~d}, J_{\mathrm{CP}}=7,2 \times \mathrm{CO}\right), 112.1\left[\mathrm{~s},{ }^{2,6} \mathrm{C}\left(\mathrm{C}_{6} \mathrm{H}_{2}\right)\right]$, $99.3\left[\mathrm{~s},{ }^{4} \mathrm{C}\left(\mathrm{C}_{6} \mathrm{H}_{2}\right)\right], 92.5,88.0(2 \times \mathrm{s}, 2 \times \mathrm{Cp}), 84.2\left[\mathrm{~d}, J_{\mathrm{CP}}=74\right.$, $\left.{ }^{1} \mathrm{C}\left(\mathrm{C}_{6} \mathrm{H}_{2}\right)\right], 80.2\left[\mathrm{~s},{ }^{3,5} \mathrm{C}\left(\mathrm{C}_{6} \mathrm{H}_{2}\right)\right], 35.7\left[\mathrm{~s}, 2 \mathrm{x}{ }^{2} \mathrm{C}\left({ }^{\mathrm{t}} \mathrm{Bu}\right)\right], 35.1[\mathrm{~s}, 2 \mathrm{x}$ $\left.{ }^{1} \mathrm{C}\left({ }^{\mathrm{B}} \mathrm{Bu}\right)\right], 34.5\left[\mathrm{~s},{ }^{1} \mathrm{C}\left({ }^{\mathrm{t}} \mathrm{Bu}\right)\right], 31.3\left[\mathrm{~s},{ }^{2} \mathrm{C}\left({ }^{\mathrm{t}} \mathrm{Bu}\right)\right]$.

Preparation of $\left[\mathrm{Mo}_{2} \mathrm{Cp}_{2}\left(\mu-\kappa^{1}: \kappa^{1}, \eta^{4}-\mathbf{P R}^{*}\right)(\mathrm{CO})_{3}\right]$ (4). A $\mathrm{CH}_{2} \mathrm{Cl}_{2}$ solution $(10 \mathrm{~mL})$ of compound $3(0.030 \mathrm{~g}, 0.046 \mathrm{mmol})$ was placed in a bulb equipped with a Young's valve. The bulb was cooled at $77 \mathrm{~K}$, evacuated and then refilled with CO. The valve was then closed and the solution was allowed to reach room temperature and further stirred for 72 h. Solvent was then removed in vacuo and the residue was dissolved in a minimum of toluene and chromatographed on alumina (activity IV, $30 \mathrm{x}$ $3 \mathrm{~cm})$ at $288 \mathrm{~K}$. Elution with petroleum ether gave a black fraction. Removal of solvents from the latter yielded $\left[\mathrm{Mo}_{2} \mathrm{Cp}_{2}\left(\mu-\mathrm{PR}^{*}\right)(\mathrm{CO})_{4}\right]$ $(0.005 \mathrm{~g}, 15 \%)$ as a black microcrystalline solid. Elution with dichloromethane:petroleum ether (1:1) gave a yellow fraction. Removal of solvent from this fraction gave compound $4(0.018 \mathrm{~g}, 58 \%)$ as an orange microcrystalline solid. Analysis Calculated for $\mathrm{C}_{31} \mathrm{H}_{39} \mathrm{Mo}_{2} \mathrm{O}_{3} \mathrm{P}(4)$ : 
C, 54.22; H, 5.73. Found: C, 54.38; H, 5.79. $v_{\mathrm{CO}}\left(\mathrm{CH}_{2} \mathrm{Cl}_{2}\right): 1961$ (s), 1899 (vs), 1823 (s) $\mathrm{cm}^{-1} .{ }^{1} \mathrm{H}$ NMR: $\delta 6.19,5.74\left(2 \times \mathrm{xs}, 2 \times 1 \mathrm{H}, \mathrm{C}_{6} \mathrm{H}_{2}\right)$, 5.51, $5.45(2 \times \mathrm{s}, 2 \times 5 \mathrm{H}, \mathrm{Cp}), 1.43,1.13,1.03$ (3 x s, 3 x 9H, $\left.{ }^{\mathrm{t}} \mathrm{Bu}\right)$. ${ }^{31} \mathrm{P}\left\{{ }^{1} \mathrm{H}\right\}$ NMR: $\delta 476.1$ (s, $\mu$-PR*). ${ }^{13} \mathrm{C}\left\{{ }^{1} \mathrm{H}\right\}$ NMR: $\delta 243.9\left(\mathrm{~d}, J_{\mathrm{CP}}=16\right.$, $\mathrm{CO}), 238.9\left(\mathrm{~d}, J_{\mathrm{CP}}=17, \mathrm{CO}\right), 234.5\left(\mathrm{~d}, J_{\mathrm{CP}}=13, \mathrm{CO}\right), 158.5[\mathrm{~s}$, $\left.{ }^{6} \mathrm{C}\left(\mathrm{C}_{6} \mathrm{H}_{2}\right)\right], 126.3\left[\mathrm{~s},{ }^{5} \mathrm{C}\left(\mathrm{C}_{6} \mathrm{H}_{2}\right)\right], 109.8,108.9\left[\mathrm{~s},{ }^{2} \mathrm{C}\right.$ and $\left.{ }^{4} \mathrm{C}\left(\mathrm{C}_{6} \mathrm{H}_{2}\right)\right], 91.7$, $91.4(2 \times \mathrm{s}, 2 \times \mathrm{Cp}), 87.2\left[\mathrm{~s},{ }^{3} \mathrm{C}\left(\mathrm{C}_{6} \mathrm{H}_{2}\right)\right], 78.8\left[\mathrm{~d}, J_{\mathrm{CP}}=65,{ }^{1} \mathrm{C}\left(\mathrm{C}_{6} \mathrm{H}_{2}\right)\right]$, 39.9, 35.3, 35.2 [3 x s, $\left.{ }^{1} \mathrm{C}\left({ }^{\mathrm{t}} \mathrm{Bu}\right)\right], 33.7,31.7,30.3\left[3 \mathrm{x} \mathrm{s},{ }^{2} \mathrm{C}\left({ }^{\mathrm{t}} \mathrm{Bu}\right)\right]$.

Preparation of solutions of $\left[\mathrm{Mo}_{2} \mathrm{Cp}_{2}(\mathrm{H})\left(\mu-\kappa^{1}: \kappa^{1}, \eta^{6}-\right.\right.$ $\left.\left.\mathbf{P R}^{*}\right)(\mathbf{C O})_{2}\right]\left[\mathbf{B A r}_{4}{ }_{4}\right](5)$. A mixture of compound $3(0.025 \mathrm{~g}, 0.038 \mathrm{mmol})$ and $\left[\mathrm{H}\left(\mathrm{OEt}_{2}\right)_{2}\right]\left[\mathrm{BAr}_{4}{ }_{4}\right](0.036 \mathrm{~g}, 0.038 \mathrm{mmol})$ were dissolved at $233 \mathrm{~K}$ in $\mathrm{CD}_{2} \mathrm{Cl}_{2}(0.8 \mathrm{~mL})$. The mixture was stirred for $5 \mathrm{~min}$ and then transferred into an NMR tube kept at the same temperature. NMR data on this red solution showed complete conversion of compound $\mathbf{3}$ into the hydride derivative $\mathbf{5}$, which in solution exists as two isomers which rapidly interconvert on the NMR time scale at ambient temperature. Complete data could only be obtained for the major (trans) isomer. $v_{\mathrm{CO}}\left(\mathrm{CH}_{2} \mathrm{Cl}_{2}\right)$ : 1986 (s), 1932 (vs) $\mathrm{cm}^{-1} .{ }^{1} \mathrm{H}$ NMR $(200.13 \mathrm{MHz}, 291 \mathrm{~K}$, averaged spectrum): $\delta 7.72\left(\mathrm{~s}, \mathrm{br}, 8 \mathrm{H}, \mathrm{Ar}^{\prime}\right), 7.56(\mathrm{~s}, \mathrm{br}, 4 \mathrm{H}, \mathrm{Ar}$ ), $6.01(\mathrm{~s}, 2 \mathrm{H}$, $\left.\mathrm{C}_{6} \mathrm{H}_{2}\right), 5.65(\mathrm{~s}, 5 \mathrm{H}, \mathrm{Cp}), 5.48\left(\mathrm{~d}, J_{\mathrm{HP}}=3,5 \mathrm{H}, \mathrm{Cp}\right), 1.27\left(\mathrm{~s}, 18 \mathrm{H}, 2 \times{ }^{\mathrm{t}} \mathrm{Bu}\right)$, $1.25\left(\mathrm{~s}, 9 \mathrm{H},{ }^{\mathrm{t}} \mathrm{Bu}\right),-3.62\left(\mathrm{~d}, J_{\mathrm{HP}}=45,1 \mathrm{H}, \mathrm{Mo}-\mathrm{H}\right) .{ }^{31} \mathrm{P}\left\{{ }^{1} \mathrm{H}\right\}$ NMR $(291 \mathrm{~K}$, averaged spectrum): $\delta 643.0\left(\mathrm{~s}, \mu-\mathrm{PR}^{*}\right)$. Data for trans-5: ${ }^{1} \mathrm{H}$ NMR (400.13MHz, $213 \mathrm{~K}): \delta 7.81$ (s, br, 8H, Ar'), 7.62 (s, br, 4H, Ar'), 5.99 (s, $\left.2 \mathrm{H}, \mathrm{C}_{6} \mathrm{H}_{2}\right), 5.67,5.52(2 \times \mathrm{s}, 2 \times 5 \mathrm{H}, \mathrm{Cp}), 1.25\left(\mathrm{~s}, 18 \mathrm{H}, 2 \times{ }^{\mathrm{t}} \mathrm{Bu}\right), 1.23(\mathrm{~s}$, $\left.9 \mathrm{H},{ }^{\mathrm{t}} \mathrm{Bu}\right),-3.91\left(\mathrm{~d}, J_{\mathrm{HP}}=20,1 \mathrm{H}, \mathrm{Mo}-\mathrm{H}\right) .{ }^{31} \mathrm{P}\left\{{ }^{\mathrm{l}} \mathrm{H}\right\}$ NMR $(162.09 \mathrm{MHz}$, $213 \mathrm{~K}): \delta 644.6\left(\mathrm{~s}, \mu\right.$-PR*). Data for cis-5: ${ }^{1} \mathrm{H}$ NMR $(400.13 \mathrm{MHz}$, 213K): $\delta-2.52$ (br, $1 \mathrm{H}, \mathrm{Mo}-\mathrm{H})$. The cis/trans ratios measured from the integrals of the hydride resonances were 0.1 at $213 \mathrm{~K}$ and 0.16 at $243 \mathrm{~K}$. This ratio was calculated to be 1.4 at $291 \mathrm{~K}$.

Preparation of $\left[\mathrm{Mo}_{2} \mathrm{Cp}_{2}(\mu-\mathrm{P})\left(\eta^{6}-\mathrm{C}_{6} \mathrm{H}_{3}{ }^{\mathrm{t}} \mathrm{Bu}_{3}\right)(\mathrm{CO})_{2}\right]\left[\mathrm{BAr}_{4}{ }_{4}\right]$ (6). A dichloromethane solution $(5 \mathrm{~mL})$ of compound $\mathbf{3}(0.025 \mathrm{~g}, 0.038 \mathrm{mmol})$ and $\left[\mathrm{H}\left(\mathrm{OEt}_{2}\right)_{2}\right]\left[\mathrm{BAr}_{4}^{\prime}\right](0.039 \mathrm{~g}, 0.041 \mathrm{mmol})$ was stirred at room temperature for $2 \mathrm{~h}$ to give a brown mixture which was filtered. Solvent was then removed from the filtrate in vacuo, and the residue washed with petroleum ether to yield compound $\mathbf{6}$ as a brown powder $(0.052 \mathrm{~g}, 90 \%)$. The crystals used in the X-ray study were grown by slow diffusion of petroleum ether into a dichloromethane solution of the compound at 253 K. Analysis Calculated for $\mathrm{C}_{62} \mathrm{H}_{52} \mathrm{BF}_{24} \mathrm{Mo}_{2} \mathrm{PO}_{2}$ (6): C, 49.03; H, 3.45 . Found: C, 49.15; H, 3.39. $v_{\mathrm{CO}}\left(\mathrm{CH}_{2} \mathrm{Cl}_{2}\right): 1987$ (vs), $1927(\mathrm{~s}) \mathrm{cm}^{-1} .{ }^{1} \mathrm{H}$ NMR (200.13 MHz): $\delta 7.72$ (s, br, 8H, Ar'), 7.56 (s, br, 4H, Ar'), 5.45 (s, $5 \mathrm{H}, \mathrm{Cp}), 5.36\left(\mathrm{~d}, J_{\mathrm{HP}}=4,3 \mathrm{H}, \eta^{6}-\mathrm{C}_{6} H_{3}{ }^{\mathrm{t}} \mathrm{Bu} 3\right), 5.11\left(\mathrm{~d}, J_{\mathrm{HP}}=3,5 \mathrm{H}, \mathrm{Cp}\right)$, $1.33\left(\mathrm{~s}, 27 \mathrm{H}, 3 \times{ }^{\mathrm{t}} \mathrm{Bu}\right) \cdot{ }^{31} \mathrm{P}\left\{{ }^{1} \mathrm{H}\right\}$ NMR: $\delta 908.5$ (s, $\left.\mu-\mathrm{P}\right) .{ }^{13} \mathrm{C}\left\{{ }^{1} \mathrm{H}\right\}$ NMR: $\delta$ $230.2(\mathrm{~s}, \mathrm{br}, 2 \times \mathrm{CO}), 162.2\left[\mathrm{q}, J_{\mathrm{CB}}=50,{ }^{1} \mathrm{C}\left(\mathrm{Ar}^{\prime}\right)\right], 135.2\left[\mathrm{~s},{ }^{2,6} \mathrm{C}\left(\mathrm{Ar}^{\prime}\right)\right]$, $129.2\left[\mathrm{q}, J_{\mathrm{CF}}=33,{ }^{3,5} \mathrm{C}\left(\mathrm{Ar}^{\prime}\right)\right], 125.0\left(\mathrm{q}, J_{\mathrm{CF}}=272,2 \times \mathrm{CF}_{3}\right), 118.7[\mathrm{~s}$,

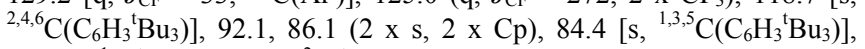
$35.8\left[\mathrm{~s},{ }^{1} \mathrm{C}\left({ }^{\mathrm{t}} \mathrm{Bu}\right)\right], 31.9\left[\mathrm{~s},{ }^{2} \mathrm{C}\left({ }^{\mathrm{t}} \mathrm{Bu}\right)\right]$.

\section{X-ray structure determination for compounds 3 and 6.}

Crystallographic data for compounds $\mathbf{3}$ and $\mathbf{6}$ are presented respectively in the CIF file format. In each case a single crystal of $\mathbf{3}$ or $\mathbf{6}$ was coated in high-vacuum grease and mounted on a glass fibre. X-ray measurements were made using a Bruker SMART CCD area-detector diffractometer with $\mathrm{Mo}-\mathrm{K}_{\alpha}$ radiation $(\lambda=0.71073 \AA)(4)$ for 3 and a Bruker Proteum diffractometer with a $\mathrm{Cu}-\mathrm{K}_{\alpha}$ radiation $(\lambda=1.541850 \AA)$ rotating anode and Osmic Confocal Maxflux mirror optics (4) for 6. Intensities were integrated (5) from several series of exposures, each exposure covering $0.3^{\circ}$ in $\omega$, and the total data set being a sphere (3) or hemisphere (6). Absorption corrections were applied, based on multiple and symmetry-equivalent measurements using SADABS (6). The structures were solved by direct methods and refined by least squares on weighted $\mathrm{F}^{2}$ values for all reflections (see Tables S1 and S6) (7). All non-hydrogen atoms were assigned anisotropic displacement parameters and refined without positional constraints. All hydrogen atoms were constrained to ideal geometries and refined with fixed isotropic displacement parameters. Refinement proceeded smoothly to give the residuals shown in Tables $\mathrm{S} 1$ and S6. Complex neutral-atom scattering factors were used ( 8$)$.

\section{References}

(1) D. D. Perrin, W. L. F. Armarego, Purification of Laboratory Chemicals. (Pergamon Press: Oxford, U.K., 1998).

(2) M. E. García, V. Riera, M. A. Ruiz, D. Sáez, J. Vaissermann, J. C. Jeffery, J. Am. Chem. Soc., 124, 14304 (2002).

(3) M. Brookhart, B. Grant, A. F. Volpe, Jr., Organometallics, 11, 3920 (1992).

(4) SMART diffractometer control software, Bruker Analytical Xray Instruments Inc., Madison, WI, 1998.

(5) SAINT integration software, Siemens Analytical X-ray Instruments Inc., Madison, WI, 1994.

(6) G. M. Sheldrick. SADABS: A program for absorption correction with the Siemens SMART system; University of Gottingen: Germany, 1996.

(7) SHELXTL program system version 5.1; Bruker Analytical X-ray Instruments Inc., Madison, WI, 1998.

(8) International Tables for Crystallography, Kluwer, Dordrecht, 1992, vol. C. 